\title{
Promotion of glycolysis by HOTAIR through GLUT1 upregulation via mTOR signaling
}

\author{
SHIBO WEI ${ }^{1}$, QING FAN ${ }^{2}$, LIANG YANG ${ }^{2}$, XIAODONG ZHANG ${ }^{2}$, YINGBO MA $^{2}$, ZHIHONG ZONG $^{3}$, \\ XIANGDONG HUA ${ }^{4}$, DONGMING SU${ }^{5}$, HONGZHI SUN ${ }^{6}, \mathrm{HANGYU} \mathrm{LI}^{2}$ and ZHEN LIU ${ }^{1}$ \\ ${ }^{1}$ Department of General Surgery, The Shengjing Hospital Affiliated to China Medical University, Shenyang, Liaoning 110004; \\ ${ }^{2}$ Department of General Surgery, The Fourth Affiliated Hospital of China Medical University, Shenyang, Liaoning 110032; \\ ${ }^{3}$ Department of Biochemistry and Molecular Biology, College of Basic Medical Sciences, China Medical University, \\ Shenyang, Liaoning 110001; ${ }^{4}$ Department of General Surgery, Liaoning Cancer Hospital and Institute, \\ Shenyang, Liaoning 110042; ${ }^{5}$ Center of Metabolic Disease Research, Nanjing Medical University, \\ Nanjing, Jiangsu 210029; ${ }^{6}$ Department of General Surgery, The First Hospital Affiliated \\ to Jinzhou Medical College, Jinzhou, Liaoning 121001, P.R. China
}

Received January 24, 2017; Accepted July 6, 2017

DOI: $10.3892 /$ or.2017.5840

\begin{abstract}
The long non-coding RNA HOX transcript antisense RNA (HOTAIR) plays a key role in the progression of various carcinomas. However, whether or not HOTAIR influences glucose metabolism and the specific underlying mechanism in hepatocellular carcinoma (HCC) cells remain unclear. In the present study, we found markedly increased HOTAIR expression in $84 \mathrm{HCC}$ tissues and demonstrated that HOTAIR overexpression promoted cell proliferation using Cell Counting Kit-8. The effect on glucose metabolism regulated by HOTAIR in HCC cells was determined by detecting lactate and glucose levels: HOTAIR promoted glycolysis by upregulating glucose transporter isoform 1 (GLUT1) and activating mammalian target of rapamycin (mTOR) signaling, whereas knockdown of HOTAIR suppressed this effect. Our research reveals a novel relationship between HOTAIR and glucose metabolism in HCC cells, and it may be a therapeutic target for diagnosing and treating HCC.
\end{abstract}

\section{Introduction}

Compared with normal cells, the majority of cancer cells generally rely on aerobic glycolysis, known as the Warburg

Correspondence to: Dr Zhen Liu, Department of General Surgery, The Shengjing Hospital Affiliated to China Medical University, 36 Sanhao Street, Shenyang, Liaoning 110004, P.R. China

E-mail: liuzhen1973@aliyun.com

Dr Hangyu Li, Department of General Surgery, The Fourth Affiliated Hospital of China Medical University, 4 Chongshan East Street, Shenyang, Liaoning 110032, P.R. China

E-mail: sj_li_hangyu@sina.com

Key words: long non-coding RNA, glycolysis, HOTAIR, GLUT1, upregulation, mTOR effect, to produce the energy required for cellular processes rather than mitochondrial oxidative phosphorylation, which is more efficient, even in the availability of oxygen (1). The reprogramming of glycometabolism not only allows the high energy demand of cancer cells to be met under hypoxic conditions, but also provides substrates for rapid synthesis of nucleotides and lipids, thereby conferring a growth advantage to cancer cells (2). Recently, the Warburg effect, or cellular energy metabolism reprogramming, was considered a new cancer biomarker (3).

MicroRNAs (miRNAs) have a crucial role in aerobic glycolysis (4). miRNAs are naturally occurring, 22-nucleotide small non-coding RNA (ncRNA) molecules (5). Recently, it was demonstrated that miR-143 has an important role in regulating glycolysis in cancer and in cancer cell proliferation; further study showed that, in lung cancer, miR-143 reduced hexokinase 2 (HK2) protein expression by targeting the mammalian target of rapamycin (mTOR) pathway (6). Another study indicated that inhibition of miR-199a, miR-138, miR-150 and miR-532-5p expression in renal cell carcinoma increased the expression of glucose transporter isoform 1 (GLUT1); conversely, increasing miR-130b, miR-19a, miR-19b and miR-301a expression downregulated GLUT1 (7). These findings demonstrate that ncRNAs may be essential regulators of aerobic glycolysis in cancer. Another indispensable ncRNA class, long ncRNAs (lncRNA) are composed of more than 200 nucleotides. To date, more than 10,000 lncRNAs have been found in the human genome (8). Several studies have found that lncRNAs can be carcinogenic or cancer-suppressor genes (9). The expression of genes involved in tumors by the comparison of tumor and normal cells has revealed abnormal lncRNA expression in different types of tumors. In addition to studies on phenotype, some in-depth studies have reported on the mechanism of lncRNAs in tumors $(10,11)$. For example, lncRNAs correlate with tumor cell apoptosis, metastasis, drug resistance and autophagy (12). Furthermore, lncRNAs have been detected in clinical routine examination of patients 
with cancer, suggesting that they can be used as a prognostic factor for diagnosing tumors. It is expected that lncRNAs may be new tumor markers and targets of tumor therapy (13). However, their role in cancer metabolism, particularly the Warburg effect, remains unknown. HOX transcript antisense RNA (HOTAIR) is the first lncRNA found to have inverse transcription, and is found in the HOXC gene on chromosome 12 (14). HOTAIR is expressed in various tumors, such as liver cancer and breast cancer, and high HOTAIR expression and poor prognosis are closely related $(15,16)$. HOTAIR expression levels were significantly positively correlated with hepatocellular carcinoma (HCC) recurrence and metastasis and with the overall survival time of patients with HCC (17). Silencing of HOTAIR expression was found to activate tumor necrosis factor (TNF)- $\alpha$-induced apoptosis, significantly decrease hepatoma cell proliferation and distant invasion ability, and also improve cancer cell sensitivity to doxorubicin and cisplatin (18). These findings suggest that HOTAIR upregulation is closely related to HCC development, metastasis, recurrence and drug resistance. Anaerobic glycolysis provides indispensable material and energy in HCC development and metastasis. Altogether, this suggests that HOTAIR may be a key role player in HCC glucose metabolism. However, the specific relationship between HOTAIR and glucose metabolism is unclear.

In the present study, we found that HOTAIR promotes HCC cell consumption of glucose and production of lactate. HOTAIR is involved in glucose metabolic processes that are essential to our understanding of tumor progression. However, the mechanism by which HOTAIR directly regulates glycolysis has not been proven. Therefore, we researched the potential mechanism of HOTAIR-mediated metabolic transformation in HCC cells.

\section{Materials and methods}

Clinical samples. From July 2012 to November 2014, 84 HCC and paired adjacent normal tissues were obtained from patients undergoing surgical hepatectomy at the Shengjing Hospital of China Medical University and who were pathologically diagnosed with HCC. None of the patients received chemotherapy or radiation therapy, preoperatively. The Shengjing Hospital Ethics Committee approved the present study; we obtained informed consent from all patients.

Cell lines and culture. HepG2, SMMC-7721, Hep3B, Huh7, and Bel-7402 cells, and the normal liver Chang cell line were purchased from the American Type Culture Collection (ATCC; Manassas, VA, USA). All cells were cultured with Dulbecco's modified Eagle's medium that contained $2 \mathrm{mM}$ L-glutamine, $0.1 \mathrm{mM}$ non-essential amino acids, $1.0 \mathrm{mM}$ sodium pyruvate, and $10 \%$ fetal bovine serum in $5 \% \mathrm{CO}_{2}$ and $95 \% \mathrm{O}_{2}$ at $37^{\circ} \mathrm{C}$.

Lactate and glucose level measurement. Lactate and glucose levels were detected using Lactate Assay Kit II and High Sensitivity Glucose Assay kit, respectively. The two kits are purchased from Sigma-Aldrich, Shanghai, China.

Cell Counting Kit-8 (CCK-8). CCK-8 (Beyotime) was used to detect cell proliferation. Cells $\left(5 \times 10^{3}\right.$ cells $\left./ \mathrm{ml}\right)$ were seeded in 96-well plates, incubated in $5 \% \mathrm{CO}_{2}$ overnight at $37^{\circ} \mathrm{C}$, and then incubated for $1 \mathrm{~h}$ with $10 \mu \mathrm{l} \mathrm{CCK}-8$ reagent. We measured the CCK-8 absorption values (A) at $450 \mathrm{~nm}$ using a microplate reader to determine the rate of cell growth.

Western blotting. Western blot assays were performed as previously described (12). Anti-GLUT1 and anti-phosphorylated (p)-mTOR rabbit monoclonal antibody and anti-immunoglobulin (Ig)G rabbit monoclonal antibody were purchased from ProteinTech Group (Chicago, IL, USA). We purchased horseradish peroxidase-linked secondary antibodies from Cell Signaling Technology (Shanghai, China). Anti- $\beta$-actin was purchased from TransGen Biotech (Beijing, China).

Real-time PCR. Isolation of total RNA, reverse transcription of RNA, and quantitative real-time PCR were performed as previously described (12) using the following forward and reverse primers: HOTAIR, 5'-CAGTGGGGAACTCT GACTCG-3' and 5'-GTGCCTGGTGCTCTCTTACC-3'; GLUT1, 5'-TTATTGCCCAGGTGTTCGGC-3' and 5'-GTAGCAGGGCTGGGATGAAG-3'; 18S, 5'-GGAGCGAG ATCCCTCCAAAAT-3' and 5'-GGCTGTTGTCATACTTCTC ATGG-3'.

RNA binding protein immunoprecipitation (RIP). A RIP kit was purchased from Millipore (Bedford, MA, USA); the RIP assays were performed according to the manufacturer's protocol. The negative control and positive control were normal rabbit IgG and GLUT1, respectively. The co-precipitated RNAs were determined by quantitative reverse transcription-PCR (qRT-PCR).

HOTAIR small interfering RNA (siRNA) vector construction and transfection. We purchased plasmids that contained HOTAIR complementary DNA (cDNA), HOTAIR siRNA (si-HOTAIR) and control siRNA from GeneChem (Shanghai, China), and transfected them into cells using Lipofectamine 2000 according to the manufacturer's instructions.

Statistical analysis. We analyzed the data using SPSS 17.0 software (SPSS, Inc., Chicago, IL, USA). Data are presented as the mean \pm standard deviation (SD). The Student's t-test or analysis of variance was used for the statistical analyses. $\mathrm{P}<0.05$ was considered statistically significant.

\section{Results}

HOTAIR expression is high in HCC cells and tissues. Real-time PCR was used to investigate HOTAIR expression levels in HCC tissues: HOTAIR expression in the tumor samples was significantly upregulated as compared to the normal tissue samples $(\mathrm{P}<0.01$; Fig. 1A), and HOTAIR expression was higher in 57 of the 84 HCC tissues (Fig. 1B). We also detected HOTAIR expression levels in HepG2, Bel-7402, Huh7, Hep3B and SMMC-7721 cell lines and in Chang cells. Real-time PCR confirmed HOTAIR upregulation in all HCC cell lines except Hep3B cells (Fig. 1C). Accordingly, we used Hep3B and HepG2 cells in the present study. 
A

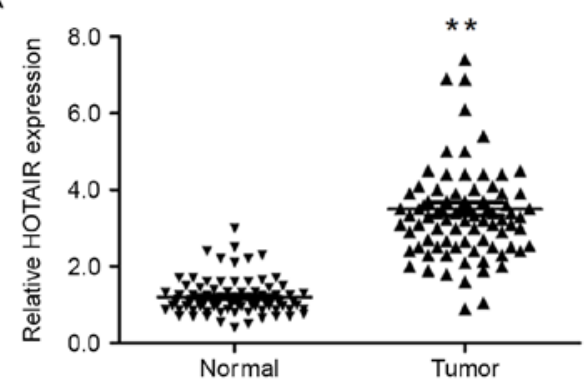

B

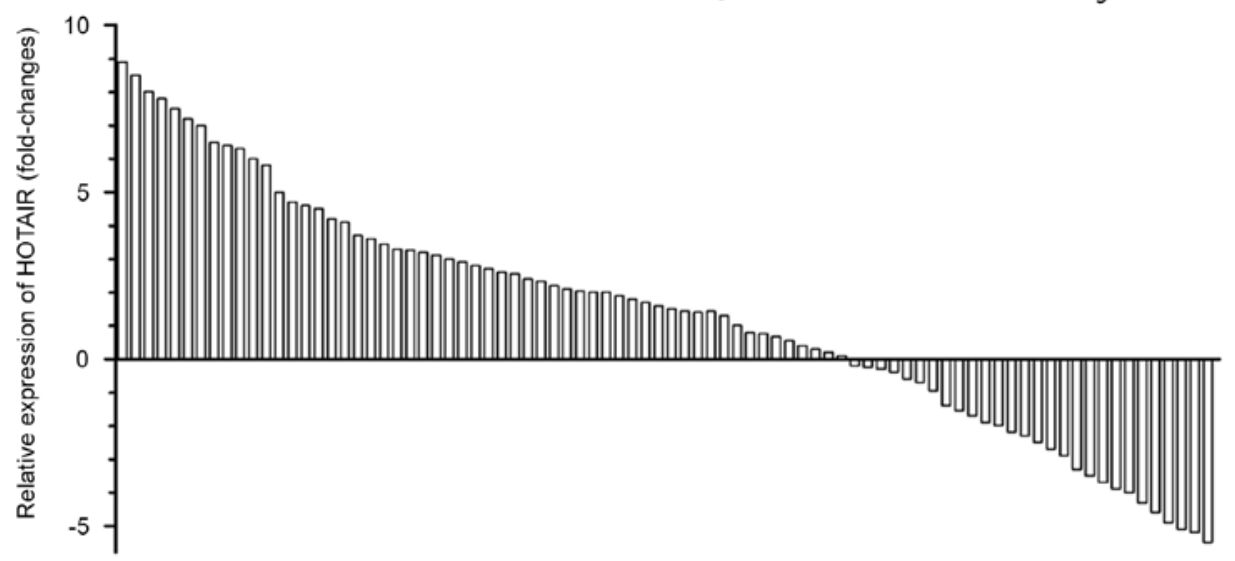

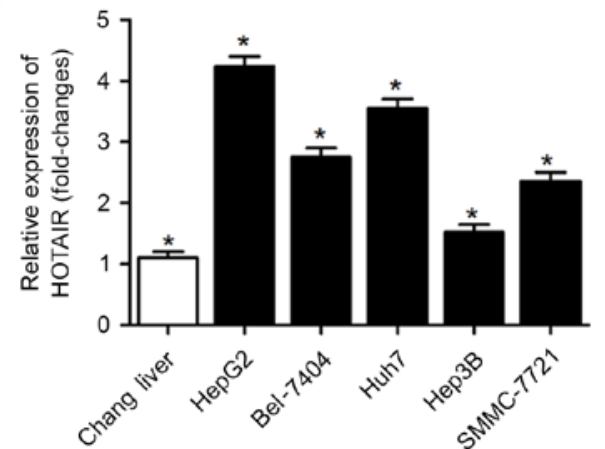

Figure 1. HOTAIR expression is high in HCC cells and tissues. (A) Real-time PCR detection of HOTAIR expression levels in HCC tissues ( $\mathrm{n}=84$ ) and paired adjacent normal samples $(\mathrm{n}=84)$. HOTAIR was significantly upregulated in the tumor tissues. (B) HOTAIR expression levels in tumor tissues. (C) HOTAIR expression in HCC cell lines (HepG2, Bel-7402, Huh7, Hep3B and SMMC-7721), and Chang cell line. Data shown are the mean \pm SD; ${ }^{*} \mathrm{P}<0.05$, ${ }^{* * *} \mathrm{P}<0.01$.

A
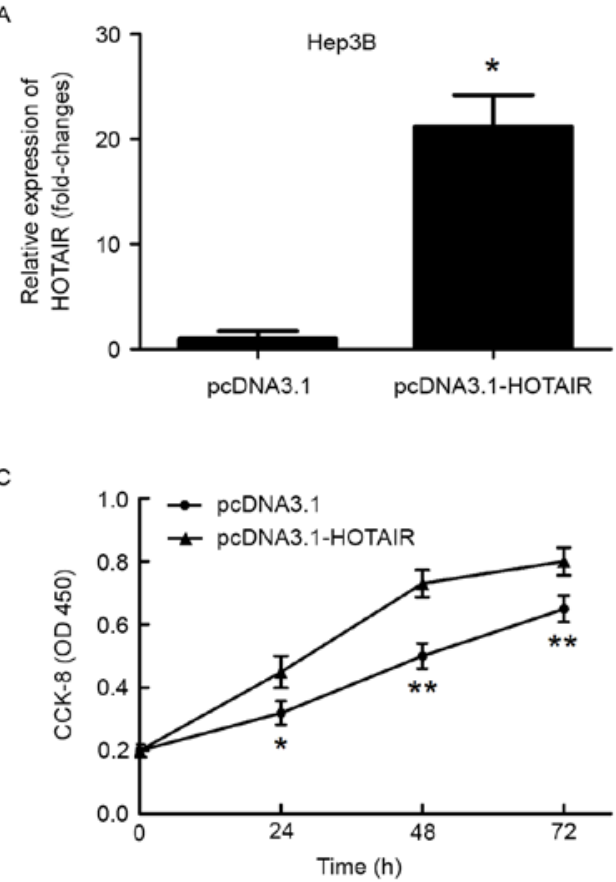
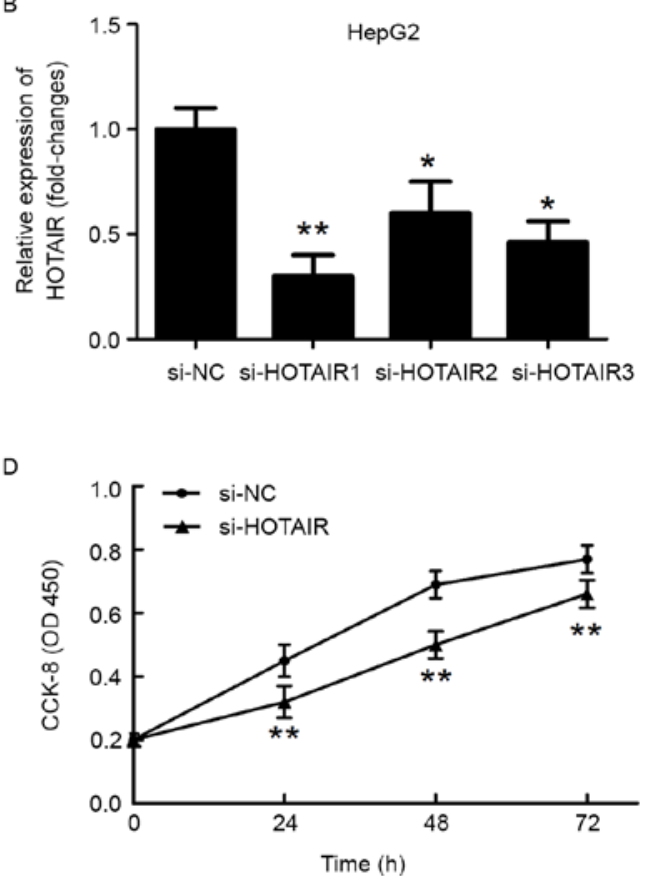

Figure 2. HOTAIR overexpression promotes in vitro HCC cell proliferation. (A) Real-time PCR analysis of HOTAIR expression in Hep3B cells following pCDNA3.1 or pcDNA3.1-HOTAIR transfection. (B) Real-time PCR analysis of HOTAIR expression in HepG2 cells following si-NC or si-HOTAIR transfection. (C) CCK-8 assay evaluation of Hep3B cell proliferation following pCDNA3.1-HOTAIR transfection. (D) CCK-8 detection of HepG2 cell proliferation following si-HOTAIR transfection. Data shown are the mean $\pm \mathrm{SD} ;{ }^{*} \mathrm{P}<0.05,{ }^{* * *} \mathrm{P}<0.01$. si-NC, negative control.

HOTAIR overexpression promotes in vitro proliferation of HCC cells. To evaluate the role of HOTAIR in HCC cell proliferation, we first transfected si-HOTAIR into HepG2 cells to decrease HOTAIR levels, and transfected Hep3B cells with pcDNA3.1-HOTAIR to upregulate HOTAIR levels. After a 48-h transfection, HOTAIR levels were 
A

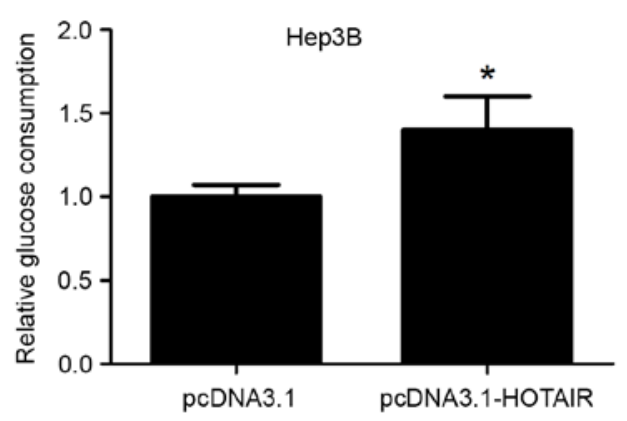

C

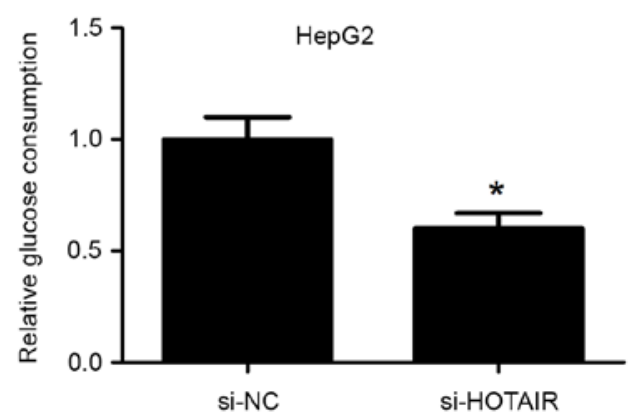

B

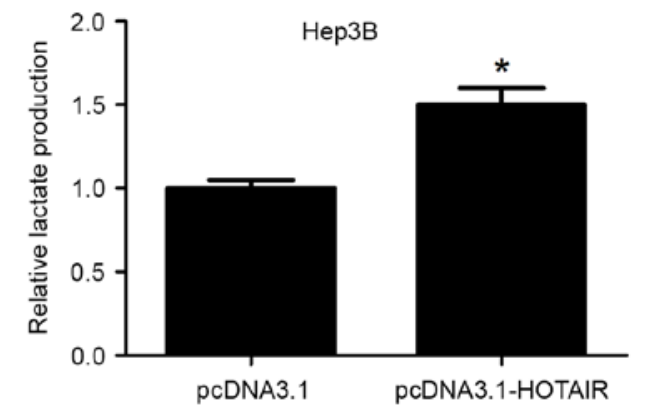

D

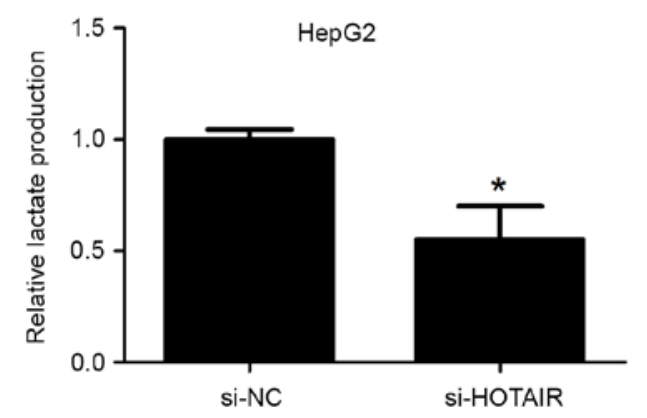

Figure 3. HOTAIR promotes glycolysis in HCC cells. Analysis of the consumption of glucose and production of lactate in (A and B) Hep3B cells with HOTAIR overexpression and in $(C$ and $D)$ HepG2 cells with HOTAIR knockdown. Data shown are the mean \pm SD; ${ }^{*} \mathrm{P}<0.05$.

measured using real-time PCR to evaluate transfection efficiency (Fig. 2A and B). CCK-8 assay showed that HOTAIR overexpression significantly promoted Hep3B cell growth as compared with the vector control (Fig. 2C). HCC cell proliferation was decreased following HOTAIR knockdown (Fig. 2D).

HOTAIR promotes glycolysis in HCC cells. Cancer cells usually utilize energy metabolism, such as glycolysis, to survive and proliferate. Therefore, we aimed to ascertain whether HOTAIR promotes cancer cell glycolysis. We first examined the effect of HOTAIR on HCC cell glucose metabolism. HOTAIR overexpression significantly increased Hep3B cell glucose consumption and lactate production rates (Fig. 3A and B), while HOTAIR knockdown in HepG2 cells significantly decreased glucose consumption and lactate production (Fig. 3C and D). The findings indicate that HOTAIR promotes glycolysis in HCC cells.

HOTAIR upregulates GLUT1 to promote glycolysis. Aerobic glycolysis involves numerous enzymes. To elucidate the potential HOTAIR regulatory mechanism of HCC cell glycolysis, we investigated the expression levels of key enzymes following HOTAIR upregulation. Real-time PCR showed that HOTAIR significantly upregulated GLUT1 mRNA levels (Fig. 4A). Western blotting showed that HOTAIR enhanced GLUT1 protein expression, while HOTAIR knockdown significantly reduced GLUT1 protein levels (Fig. 4B). In addition, GLUT1 knockdown significantly decreased the effect of HOTAIR on the consumption of glucose and production of lactate (Fig. 4C). These results indicate that HOTAIR regulates glycolysis via GLUT1.
HOTAIR promotes HCC cell glycolysis partly by inducing GLUT1 expression by upregulating mTOR and by binding GLUT1 directly. The role of mTOR in the regulation of cell metabolism is crucial. Accordingly, we detected mTOR protein expression using western blotting. There was a positive relation between mTOR phosphorylation and HOTAIR in HCC cells (Fig. 5A and B), and rapamycin reduced GLUT1 mRNA levels (Fig. 5C). We also found that rapamycin significantly decreased the glucose consumption and lactate production rates (Fig. 5D and E). Furthermore, lncRNA can also bind proteins directly, thus we used RIP to detect the interaction between HOTAIR and GLUT1, and HOTAIR bound with GLUT1 directly (Fig. 5F). Taken together, the results suggest that HOTAIR promotes HCC cell glycolysis partly by inducing GLUT1 expression by upregulating mTOR, and by binding with GLUT1 directly.

\section{Discussion}

Previously, ncRNAs were considered transcriptional noise since they did not encode proteins. However, completion of the Human Genome Project has changed this viewpoint (19). An increasing number of studies have confirmed that ncRNAs play an important regulatory role in many cellular activities and human disease, including cancer (20). In recent years, it has been reported that many lncRNAs influence the malignant biological behavior in tumor development, such as proliferation, apoptosis, invasion and metastasis (21-23). More attention has been focused on the role of lncRNAs in cancer cell development; however, the relationship between lncRNAs and cancer cell glucose metabolism remains unclear. An increasing number of studies suggest that altered metabolism 
A

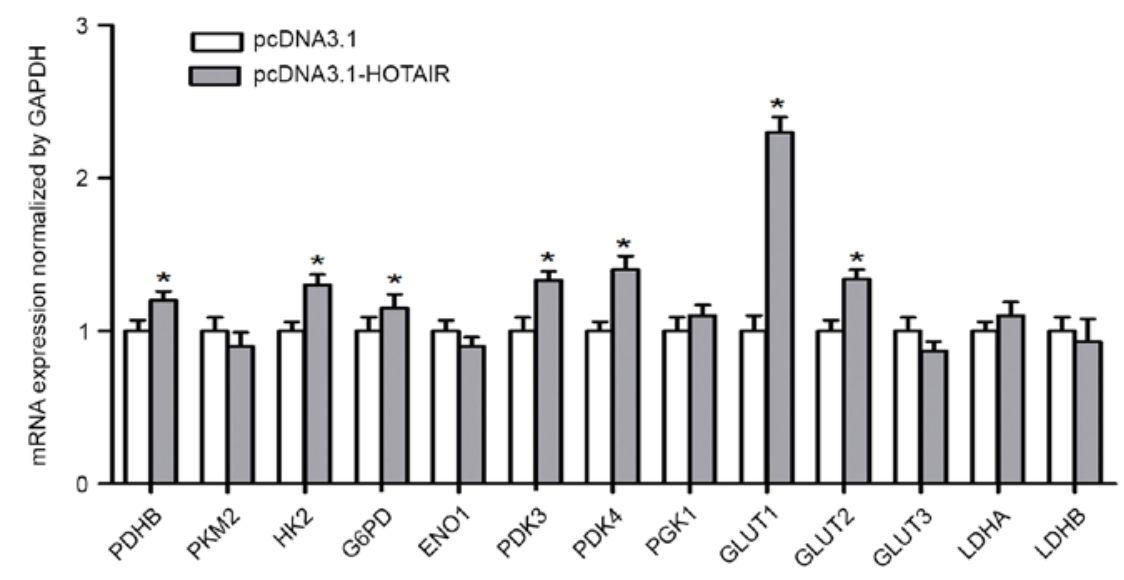

B
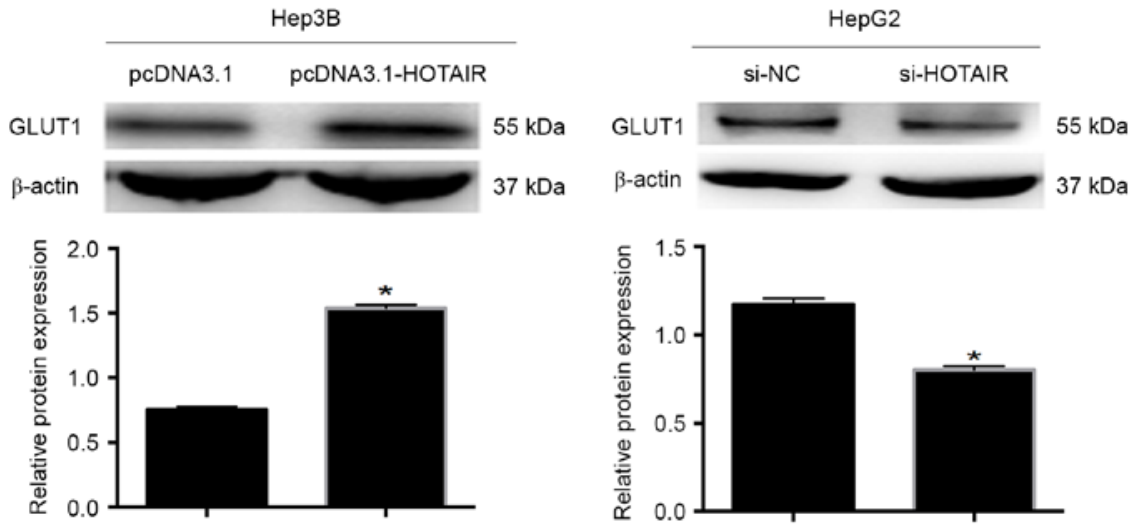

$\mathrm{C}$
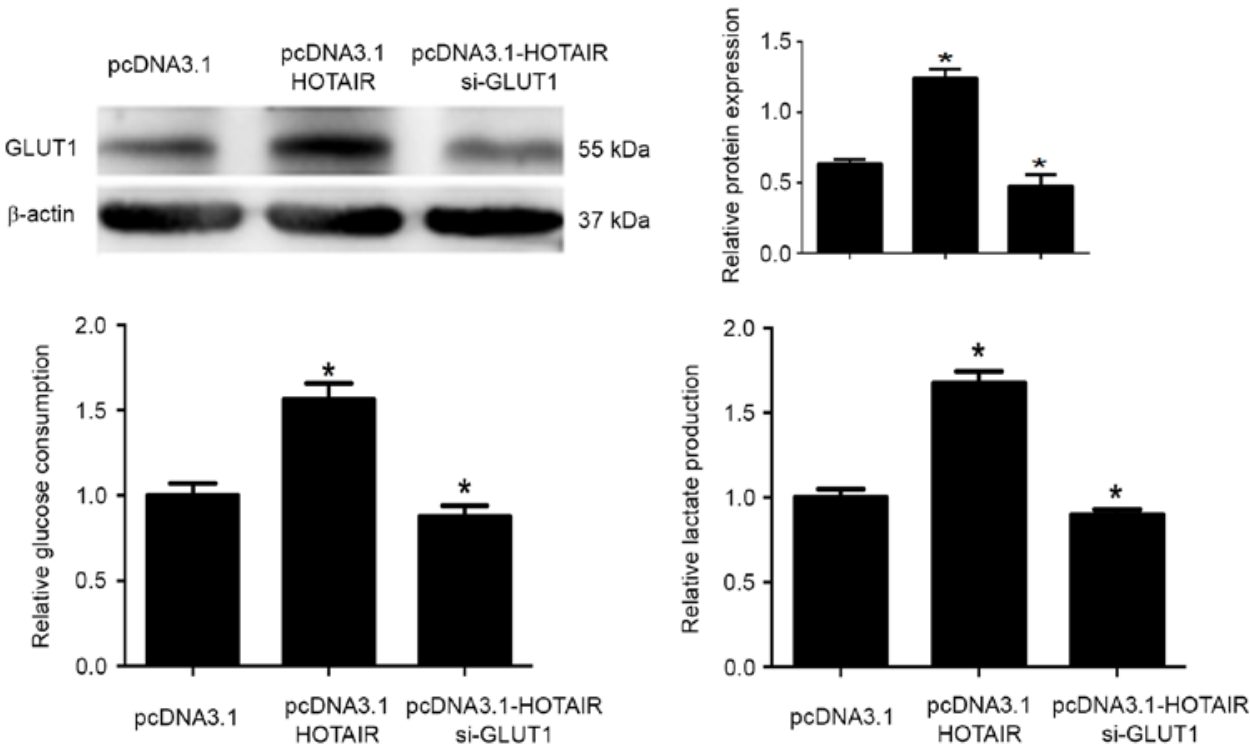

Figure 4. HOTAIR upregulates GLUT1 to promote glycolysis. (A) Real-time PCR analysis of the expression of key enzymes in Hep3B cells overexpressing HOTAIR. (B) Western blot analysis of GLUT1 protein expression in Hep3B cells overexpressing HOTAIR or in HepG2 cells with HOTAIR knockdown. (C) Analysis of the consumption of glucose and production of lactate in Hep3B cells overexpressing HOTAIR or in Hep3B cells overexpressing HOTAIR and si-GLUT1. Data shown are the mean $\pm \mathrm{SD}$; $\mathrm{P}<0.05$. si-GLUT1, GLUT1 siRNA.

is important in supporting cancer cell proliferation (24). In the present study, we demonstrated that HOTAIR is essential for accelerating glycolysis in HCC cells, which supplements the function of HOTAIR in tumor cell development.

RinnetalfirstdescribedHOTAIR as aspliced,polyadenylated ncRNA containing 2,158 nucleotides and 6 exons (25). Transcription of the $H O X C$ gene antisense strand, situated between $H O X C 11$ and $H O X C 12$ on chromosome 12q13.13, yields HOTAIR. HOTAIR interacts with polycomb repressive complex 2 (PRC2), which is necessary for PRC2 occupancy and histone H3 lysine 27 trimethylation (26). As an oncogenic factor, HOTAIR has been detected with deregulation in many cancers, such as breast and gastric cancer $(27,28)$. In the present study, 57 tumor samples $(57 / 84,68 \%)$ had higher HOTAIR 

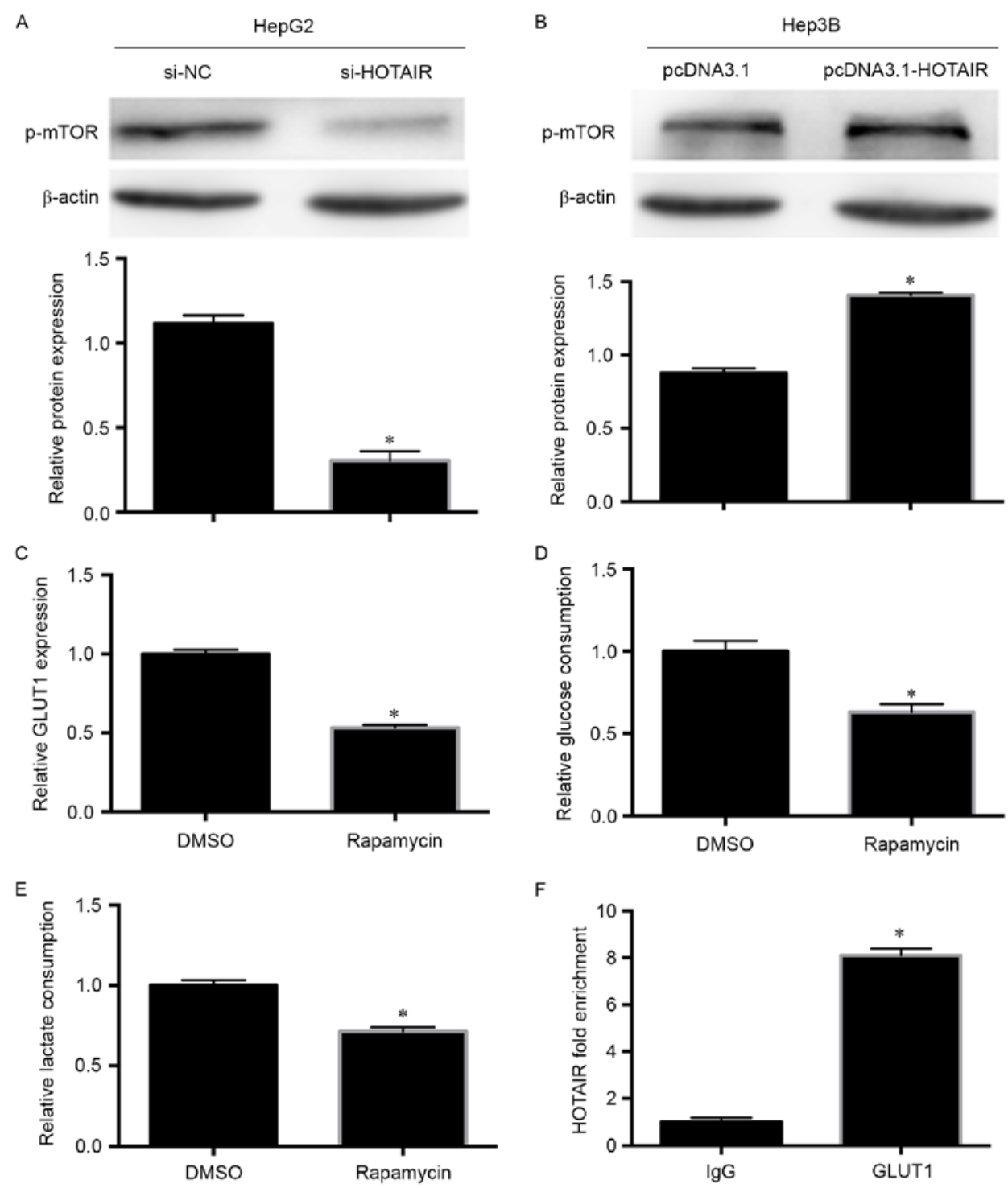

Figure 5. HOTAIR promotes glycolysis in HCC cells partly by inducing GLUT1 expression via mTOR upregulation, and by binding with GLUT1 directly. (A and B) Western blot analysis of p-mTOR protein expression in HepG2 cells with HOTAIR knockdown or in Hep3B cells overexpressing HOTAIR. (C) Real-time PCR analysis of GLUT1 expression in Hep3B cells treated with rapamycin or dimethyl sulfoxide (DMSO). (D and E) Analysis of the consumption of glucose and production of lactate in rapamycin- or DMSO-treated Hep3B cells. (F) RIP analysis of the interaction between HOTAIR and GLUT1. Data shown are the mean $\pm \mathrm{SD} ;{ }^{*} \mathrm{P}<0.05$.

expression than the matched adjacent normal tissue, which is consistent with other results on HOTAIR gene expression in HCC (18). In addition, numerous studies have focused on the effect of HOTAIR on HCC development, a complex process associated with malignant biology. Gao et al found that HOTAIR promotes cell proliferation and invasion partly by regulating the $\mathrm{Wnt} / \beta$-catenin signaling pathway (29). Ding et al demonstrated that HOTAIR increases HCC cell aggression and invasion by suppressing RBM38 expression, which plays a role in regulating cell motility (30). Recently, emerging evidence has demonstrated that HOTAIR mediates hepatocarcinogenesis by decreasing miR-128 expression, and then activating P14 and P16 signaling, functioning as a competitive endogenous RNA (ceRNA) (31). Previously, our research indicated that HOTAIR promotes autophagy activation in HCC cells by upregulating the expression of ATG3 and ATG7, autophagyrelated genes, highlighting the novel role of HOTAIR in HCC cell progression (12). However, an increasing number of studies have focused on the mechanisms of HOTAIR regulation in cancer cells, but it is still limited to research on the relationship between metabolism reprogramming in cancer cells and HOTAIR.

It was recently suggested that the lncRNA CRNDE, controlled by insulin/insulin-like growth factor (IGF) signaling, promotes cellular metabolism in a pattern that suggests the Warburg effect (32). The lncRNA UCA1 promotes glycolysis by upregulating HK2 via the mTORsignal transducer and activator of transcription (STAT)3/ miR-143 pathway (33). However, whether HOTAIR regulates HCC cell glucose metabolism remains unknown. In the present study, we confirmed that HOTAIR promotes glycolysis significantly in HCC cells, indicating that HOTAIR is involved in the metabolic regulatory process in cancer. As lncRNAs mainly function as key regulators of gene expression, we used qRT-PCR to screen a series of genes related to glucose metabolism in cells overexpressing HOTAIR as 
compared with negative control cells. HOTAIR upregulated GLUT1 most obviously at both the mRNA and protein levels. GLUT1 is an important rate-limiting factor in glucose transport and metabolism in cancer cells (34). Amann et al found significantly higher GLUT1 expression in HCC tissues which functionally affected HCC cell proliferation and invasiveness (35). Mechanistically, we showed that HOTAIR increased GLUT1 expression by upregulating mTOR and by binding GLUT1 directly. IncRNAs, such as H19 and Linc00152, are gene enhancers by direct binding with proteins $(36,37)$. Therefore, the present study reveals a novel HOTAIR/mTOR/ GLUT1 axis linking HOTAIR and glucose metabolism in HCC cells.

To summarize, HOTAIR is increased in HCC tissues and promotes cell proliferation by regulating glucose metabolism via the activation of mTOR signaling. The identification of GLUT1 as a HOTAIR-regulated gene provides a potential link between HOTAIR and cancer metabolism. However, additional mechanisms underlying the regulation between HOTAIR and metabolism warrant further exploration. The present study identifies a potential target for developing HCC therapeutic strategies.

\section{References}

1. Warburg O: On the origin of cancer cells. Science 123: 309-314, 1956.

2. DeBerardinis RJ and Thompson CB: Cellular metabolism and disease: What do metabolic outliers teach us? Cell 148: 1132-1144, 2012.

3. Munkley J and Elliott DJ: Hallmarks of glycosylation in cancer. Oncotarget 7: 35478-35489, 2016.

4. Bensinger SJ and Christofk HR: New aspects of the Warburg effect in cancer cell biology. Semin Cell Dev Biol 23: 352-361, 2012.

5. Yin S, Fan Y, Zhang H, Zhao Z, Hao Y, Li J, Sun C, Yang J, Yang $\mathrm{Z}$, Yang $\mathrm{X}$, et al: Differential TGF $\beta$ pathway targeting by miR-122 in humans and mice affects liver cancer metastasis. Nat Commun 7: 11012, 2016.

6. Fang R, Xiao T, Fang Z, Sun Y, Li F, Gao Y, Feng Y, Li L, Wang Y, Liu X, et al: MicroRNA-143 (miR-143) regulates cancer glycolysis via targeting hexokinase 2 gene. J Biol Chem 287: 23227-23235, 2012 .

7. Koh HJ, Toyoda T, Fujii N, Jung MM, Rathod A, Middelbeek RJ, Lessard SJ, Treebak JT, Tsuchihara K, Esumi H, et al: Sucrose nonfermenting AMPK-related kinase (SNARK) mediates contraction-stimulated glucose transport in mouse skeletal muscle. Proc Natl Acad Sci USA 107: 15541-15546, 2010.

8. Zhao XY and Lin JD: Long Non-coding RNAs: A new regulatory code in metabolic control. Trends Biochem Sci 40: 586-596, 2015.

9. Schmitt AM and Chang HY: Long non-coding RNAs in cancer pathways. Cancer Cell 29: 452-463, 2016.

10. Iyer MK, Niknafs YS, Malik R, Singhal U, Sahu A, Hosono Y, Barrette TR, Prensner JR, Evans JR, Zhao S, et al: The landscape of long non-coding RNAs in the human transcriptome. Nat Genet 47: 199-208, 2015.

11. Martens-Uzunova ES, Böttcher R, Croce CM, Jenster G, Visakorpi $\mathrm{T}$ and Calin GA: Long non-coding RNA in prostate, bladder, and kidney cancer. Eur Urol 65: 1140-1151, 2014.

12. Yang L, Zhang X, Li H and Liu J: The long non-coding RNA HOTAIR activates autophagy by upregulating ATG3 and ATG7 in hepatocellular carcinoma. Mol Biosyst 12: 2605-2612, 2016.

13. Ma Y, Huang D, Yang F, Tian M, Wang Y, Shen D, Wang Q, Chen $\mathrm{Q}$ and Zhang L: Long non-coding RNA highly upregulated in liver cancer regulates the tumor necrosis factor- $\alpha$-induced apoptosis in human vascular endothelial cells. DNA Cell Biol 35: 296-300, 2016

14. Lee M, Kim HJ, Kim SW, Park SA, Chun KH, Cho NH, Song YS and Kim YT: The long non-coding RNA HOTAIR increases tumour growth and invasion in cervical cancer by targeting the Notch pathway. Oncotarget 7: 44558-44571, 2016.
15. Serghiou S, Kyriakopoulou A and Ioannidis JP: Long non-coding RNAs as novel predictors of survival in human cancer: A systematic review and meta-analysis. Mol Cancer 15: 50, 2016.

16. Malek E, Jagannathan S and Driscoll JJ: Correlation of long non-coding RNA expression with metastasis, drug resistance and clinical outcome in cancer. Oncotarget 5: 8027-8038, 2014.

17. Zhang J, Zhang P, Wang L, Piao HL and Ma L: Long non-coding RNA HOTAIR in carcinogenesis and metastasis. Acta Biochim Biophys Sin 46: 1-5, 2014.

18. Yang Z, Zhou L, Wu LM, Lai MC, Xie HY, Zhang F and Zheng SS: Overexpression of long non-coding RNA HOTAIR predicts tumor recurrence in hepatocellular carcinoma patients following liver transplantation. Ann Surg Oncol 18: 1243-1250, 2011.

19. Green ED, Watson JD and Collins FS: Human Genome Project: Twenty-five years of big biology. Nature 526: 29-31, 2015.

20. Evans JR, Feng FY and Chinnaiyan AM: The bright side of dark matter: lncRNAs in cancer. J Clin Invest 126: 2775-2782, 2016.

21. Li J, Huang H, Li Y, Li L, Hou W and You Z: Decreased expression of long non-coding RNA GAS5 promotes cell proliferation, migration and invasion, and indicates a poor prognosis in ovarian cancer. Oncol Rep 36: 3241-3250, 2016.

22. Jiao C, Song Z, Chen J, Zhong J, Cai W, Tian S, Chen S, Yi Y and Xiao Y: lncRNA-UCA1 enhances cell proliferation through functioning as a ceRNA of Sox4 in esophageal cancer. Oncol Rep 36: 2960-2966, 2016.

23. Xu S, Yi XM, Tang CP, Ge JP, Zhang ZY and Zhou WQ: Long non-coding RNA ATB promotes growth and epithelial-mesenchymal transition and predicts poor prognosis in human prostate carcinoma. Oncol Rep 36: 10-22, 2016.

24. Lee $\mathrm{N}$ and Kim D: Cancer metabolism: Fueling more than just growth. Mol Cells 39: 847-854, 2016.

25. Rinn JL, Kertesz M, Wang JK, Squazzo SL, Xu X, Brugmann SA, Goodnough LH, Helms JA, Farnham PJ, Segal E, et al: Functional demarcation of active and silent chromatin domains in human HOX loci by non-coding RNAs. Cell 129: 1311-1323, 2007.

26. Hajjari $\mathrm{M}$ and Salavaty A: HOTAIR: An oncogenic long non-coding RNA in different cancers. Cancer Biol Med 12: 1-9, 2015.

27. Soudyab M, Iranpour M and Ghafouri-Fard S: The role of long non-coding RNAs in breast cancer. Arch Iran Med 19: 508-517, 2016.

28. Zhao W, Dong S, Duan B, Chen P, Shi L, Gao H and Qi H: HOTAIR is a predictive and prognostic biomarker for patients with advanced gastric adenocarcinoma receiving fluorouracil and platinum combination chemotherapy. Am J Transl Res 7: 1295-1302, 2015.

29. Gao JZ, Li J, Du JL and Li XL: Long non-coding RNA HOTAIR is a marker for hepatocellular carcinoma progression and tumor recurrence. Oncol Lett 11: 1791-1798, 2016.

30. Ding C, Cheng S, Yang Z, Lv Z, Xiao H, Du C, Peng C, Xie H, Zhou L, Wu J, et al: Long non-coding RNA HOTAIR promotes cell migration and invasion via down-regulation of RNA binding motif protein 38 in hepatocellular carcinoma cells. Int J Mol Sci 15: 4060-4076, 2014.

31. Fu WM, Zhu X, Wang WM, Lu YF, Hu BG, Wang H, Liang WC, Wang SS, Ko CH, Waye MM, et al: Hotair mediates hepatocarcinogenesis through suppressing miRNA-218 expression and activating P14 and P16 signaling. J Hepatol 63: 886-895, 2015.

32. Ellis BC, Graham LD and Molloy PL: CRNDE, a long non-coding RNA responsive to insulin/IGF signaling, regulates genes involved in central metabolism. Biochim Biophys Acta 1843: 372-386, 2014

33. Li Z, Li X, Wu S, Xue M and Chen W: Long non-coding RNA UCA1 promotes glycolysis by upregulating hexokinase 2 through the mTOR-STAT3/microRNA143 pathway. Cancer Sci 105: 951-955, 2014.

34. Sun HW, Yu XJ, Wu WC, Chen J, Shi M, Zheng L and Xu J: GLUT1 and ASCT2 as predictors for prognosis of hepatocellular carcinoma. PLoS One 11: e0168907, 2016.

35. Amann T, Maegdefrau U,Hartmann A, Agaimy A, Marienhagen J, Weiss TS, Stoeltzing O, Warnecke C, Schölmerich J, Oefner PJ, et al: GLUT1 expression is increased in hepatocellular carcinoma and promotes tumorigenesis. Am J Pathol 174: 1544-1552, 2009.

36. Zhou J, Zhi X, Wang L, Wang W, Li Z, Tang J, Wang J, Zhang Q and $\mathrm{Xu} \mathrm{Z}$ : Erratum to: Linc00152 promotes proliferation in gastric cancer through the EGFR-dependent pathway. J Exp Clin Cancer Res 35: 30, 2016.

37. Li H, Yu B, Li J, Su L, Yan M, Zhu Z and Liu B: Overexpression of lncRNA H19 enhances carcinogenesis and metastasis of gastric cancer. Oncotarget 5:2318-2329, 2014. 